\section{SOBEX: A timesharing data system for sociopharmacological experiments}

\author{
DONALD F. MILLER, P. TOBIN MAGINNIS, \\ and MARVIN C. WILSON \\ University of Mississippi, Univensity, Mississippi 38677
}

We are currently examining the effects of various psychoactive drugs on the social behaviors of individuals in a group of six stumptail macaques (Macacca arctoides) (Wilson, Bellarosa, \& Bedford, 1977). Existing observational data systems were not adequate for our situation, primarily because they (1) did not permit the direct use of a system having high-speed file access, high-level languages, and sophisticated statistical packages (Fitzpatrick, 1977); (2) presented the observer with lengthy and arbitrary behavior encoding schemes (Sykes, 1977) that could not easily be learned due to the large number of behaviors we continuously sample; (3) did not permit the storage of enough data to recreate an entire observation session (Conger \& McLeod, 1977).

Our system must retrieve data pertaining to any environmental condition, any dyadic behavioral interactions (who interacts with whom), the duration of particular behavioral episodes, and the temporal relationship between any two behavioral episodes, and thus permit flexibility in the establishment of new data analysis routines. Users must be able to define new behaviors, delete old behaviors, and add observers. An encoding scheme using brief mnemonics was preferable since observers could more easily master the system, enter more behaviors per unit time, and minimize the chances of error.

SOBEX Data Logging. To begin an observation session, the observer types in a sentence to the SOBEX monitor (described below) containing the key word, "CREATE" (e.g., "Create a data module"). The SOBEX monitor then runs the SOCIAL subprogram, which requests environmental data (observer name, drug, dose, numbers of drugged animals, room temperature) from the observer and outputs the information as the first five words of the data file. After an appropriate period designed to allow the onset of the drug effect, feeding order data is requested. The number of the animal taking each of 72 monkey chows is entered in the order in which the event occurs. After the last chow is removed, the observer types a "G" to start the session.

When the SOCIAL subprogram "sees" a G command,

Send reprint requests to Donald I. Miller, Department of Phamacology, School of Pharmacy, University of Mississippi, University, Mississippi 38677. This research was supported by NIDA Grant 01764, NIII Research Support Grant PHS FRO 5594-01, and by the Research Institute of Pharmaceutical Sciences. University of Mississippi. The authors wish to acknowledge the observational assistance of Ms. Karen Lovell. it performs two tasks for the next hour: (1) It times the session, and (2) checks for three-character behavior occurrence codes. The SOBEX system allows definition of 26 behaviors (one for each letter of the alphabet). The actual names of the behaviors corresponding to each letter of the alphabet are defined in the file, BEHAVE.LST, which is read into the SOBEX monitor when the system is initialized. When a behavior occurs in the group being observed, the observer types "X12," where $\mathrm{X}$ is the one-letter behavior code, 1 is the animal number of the initiator of the behavior (actor), and 2 is the animal number of the recipient of the behavior. On the user's terminal, the text "TIME BEHAVIOR 12 " is printed; TIME is the time of day, BEHAVIOR is the behavior corresponding to the one-letter code, and 1 and 2 are the actor and recipient of the behavior. If SOCIAL is running on a cathode-ray-tube (CRT) terminal, the text is typed out in the upper left corner of the screen. Since sessions in our lab are video taped, we have merged the CRT output with the camera output; the actual behavior associated with a given code can be reviewed as necessary by replaying the video tape. The observer indicates termination of a timed behavior by typing a code such as "X10," indicating that Animal 1 stopped behavior X. At the end of $1 \mathrm{~h}$, the data module is closed. SOCIAL then creates a disk file name according to the day's date (e.g., 780129.SBX for January 29,1978 ) to receive the data from the day's session.

The data packing procedure employed by SOBEX is efficient. Each behavioral interaction is stored as one word in the data module, and, on a typical day, approximately 750 behavior interactions are entered for the 1-h session. The DECSystem-10 allocates about five disk blocks (128 36-bit words per block) for each SOBEX data module; twenty disk blocks are required for storage of the same data in ASCII format. At this rate, a user having a private 80,000 -block disk pack can store at least 25 365-day years of data modules without space problems.

We have developed an ancillary data logging program employing an LSI-11 which creates compatible SOBEX data modules. This system protects us against temporary PDP-10 failures. At the end of the session, the data is locally stored on magnetic tape and sent to the PDP- 10 .

Implementation of the SOBEX System. Interactions between the experimenter and the SOBEX system are arbitrated by the SOBEX monitor, an assembly language program that is automatically run when the user logs in on the SOBEX project area. The SOBEX monitor is designed to interpret English-like sentences and run the appropriate subprogram. Thus, the command, "List 780129 on device LPT:," produces a line printer listing of the data entered for January 29, 1978. If the user fails to give SOBEX all of the information it requires 
to run the desired subprogram, SOBEX requests the additional information from the user. Currently, the SOBEX monitor recognizes 13 key words which cause subprograms to run. Additionally, when the SOBEX monitor is initialized, it automatically logs in a subjob on the SOBEX statistical area. Commands can be passed to the TOPS monitor via the subjob, and the various DECSystem-10 resources can be utilized.

If the SOBEX system is initialized on a CRT, SOBEX assumes cursor control so that output text is presented in an orderly manner, one page at a time. While SOBEX is awaiting a command string, the time of day is displayed in the upper left comer of the CRT screen.

Statistical Analysis Procedures. Currently, three statistical procedures have been incorporated into the SOBEX monitor. SOBEX handles statistical requests as follows: When the SOBEX monitor detects a key word that requests statistics, it jumps to the appropriate subprogram. The subprogram types a message to the user on how the particular statistic will be performed (which data will be used, how the comparisons will be done, etc.). It then opens a uniquely named (e.g., SBX01, SBX02, etc.) file, with the .CTL file extension (denoting a batch job control file to TOPS) to be filled in by the SOBEX subprogram. The first text output to the SBX01.CTL file is the ASCII string ".RUN ANOVA1.EXE," or whatever the desired statistic happens to be. The subprogram then requests the dates across which analysis is desired. The entry of the last date is the signal for the subprogram to output the list of dates in ASCII to the SBX01.CTL file. The SBX01.CTL file is then closed and submitted to BATCON, the DECSystem-10 batch job controller, via the subjob link with TOPS. In this manner, a large number of statistical requests may be made and dealt with at the convenience of the local computer system.

The batch control file, when it is activated by BATCON, initiates a chain of programs that extract specified data from daily data modules and request data analysis from the Statistical Package for the Social Sciences (SPSS) (Matzek, 1977; Nie, Hull, Jenkins, Steinbrenner, \& Bent, 1975). Thus, for example, when the SOBEX program ANOVA1.EXE is run, it creates a temporary file with these instructions for SPSS: "LPT:=ANOVA1.SPS." LPT: specifies the output device, while ANOVAl.SPS indicates the input control file for SPSS. The ANOVAl.EXE program then chains to the EXTRAC program, which extracts the occurrences of the behaviors for the days named in the .CTL file. If the desired statistic requires that the behaviors of a particular actor be summed across all recipients of that behavior, the SUM program is chained next. When SUM finishes the summation process, it chains SPSS in a manner that causes it to find the temporary instruction file made by the SOBEX ANOVA1.EXE program. SPSS then finds the desired command file (in this case, ANOVA1.SPS). The command file causes SPSS to read the data using a standard SPSS subprogram. When SPSS finishes the analysis, the results are output to the line printer and the batch job is logged off the system.

The three statistical routines now incorporated into the SOBEX monitor are ANOVA1, ANOVA2, and STABIL. ANOVA1 directs the performing of a oneway analysis of variance across the desired days. Thus, in a sociopharmacological experiment in which a different dose of a drug is given on each of several days, the one-way analysis of variance indicates the impact of the drug on the summed behaviors at each dose. In this manner, the ubiquitous dose-response relationship is obtained. SPSS allows Duncan's multiple-range test, Scheffës test, or other post hoc tests to pinpoint significant differences.

ANOVA2 is similar to ANOVA1 in that a doseresponse relationship for the particular drug is described statistically. ANOVA2, however, performs a two-way analysis of variance, with day (or dose in our lab) being one dependent variable and the sex of the animal being the other dependent variable. In this way, insight can be gained into particular drugs which might affect a given behavior more in one sex than in the other.

The STABIL subprogram directs the creation of scattergrams and linear regression coefficients for each of the summed behaviors. In this manner, an estimation of the stability of the group's social interactions can be obtained. This is necessary, since the administration of a drug treatment regimen while the behaviors are dramatically changing would produce meaningless results; the changes detected could not clearly be attributed to either the drug or the changing trend.

When more statistical tests are required, they are readily incorporated into the SOBEX system by creation of appropriate .CTL files.

SOBEX Utilities. The SOBEX system includes utility subprograms that perform specific tasks designed to make life easier for the user of the system. The SUMMARY subprogram, which responds to a command string, such as "Summarize modules on LPT:," produces a listing of the environmental data for each data module in tabular form on the specified device. The subprogram is usually run prior to a statistical analysis so the desired dates can be identified.

The MODULE subprogram responds to a SOBEX monitor command, such as "List 780128 on LPT:." This subprogram is automatically engaged when running the SOCIAL subprogram results in the creation of a new data module. The MODULE subprogram creates a listing of the environmental data and each of the behavioral interactions in the specified data module.

In response to a command string incorporating a date and the key word "EXTRACT," the EXTRAC subprogram produces a listing of the specified data module, in which each behavior is expressed in terms of the occurrences of each of the possible dyadic interactions. Thus, for a given day, the sums of 936 dyadic 
interactions. are listed ( 26 behaviors $\times 6$ actors $\times 6$ recipients).

Errors are possible, so subprogram OOPS responds to a command string having a date and the key word "MISTAKE." OOPS allows the user to correct any incorrectly entered environmental data word. Corrections of social behavior interaction words are not allowed; instead, observers are advised that SOBEX analysis programs will key in on the three-letter interaction code, "X77," and thereby be instructed to ignore the previous behavioral interaction word. In this manner, there is greater certainty that the actual error, rather than a correctly entered interaction, will be removed.

Since the SOBEX monitor always uses two job slots on the DECSystem-10 (and sometimes three job slots when a batch job is also running), subprogram USERS, which responds to a command string containing the key word "SYSTAT," provides the user with a status report of SOBEX project jobs currently running.

Ordinarily, the SOBEX monitor disables the standard TOPS interrupt character (control c) so that the user is not allowed to interrupt SOBEX while leaving the subjob detached. Therefore, the SOBEX monitor responds to the command "TOPS10" to log off the subjob and return the user to the TOPS monitor.

Finally, the SOBEX key word, "LOGOFF," causes the subjob to be killed and then logs the SOBEX monitor off the system.

Limitations. SOBEX has the following limitations: (1) No more than 26 behaviors can be dealt with, (2) no more than six animals may exist in any one group, (3) only one recording session per day is allowed, and
(4) SOBEX must run on a PDP-10 computer and is, therefore, machine specific. SOBEX will, however, allow multiple observers simply by assigning each observer a different project-programmer number.

Availability. A program listing is available at no cost from Donald F. Miller, Department of Pharmacology, University of Mississippi, University, Mississippi 38677.

\section{REFERENCES}

Conger, R. D., \& McLeon, D. Describing behavior in small groups with the Datamyte recorder. Behavior Research Methods \& Instrumentation, 1977, 9, 418-424.

Digital Equipment Corporation. DECSystem-10 monitor calls manual. Maynard, Mass: Author, 1974.

Digital Equipment Corporation. DECSystem-10 MACRO10 assembler programmer's reference manual. Maynard, Mass: Author, 1975. (a)

Digital Equipment Corporation. DECSystem-10 system reference manual. Maynard, Mass: Author, 1975. (b)

FitzPatrick, L. J. BEHAVE-An automated data analysis system for observed events. Behavior Research Methods \& Instrumentation, 1977, 9, 452-455.

MATZEK, M. A. SPSS-10: Statistical package for the social sciences. (Release 7.01, documentation manual). Pittsburgh, Penn: University of Pittsburgh, October 1977.

Nie, N. H., Hull, C. H., Jenkins, J. G., Steinbrenner, K., \& BENT, D. H. SPSS: Statistical package for the social sciences (2nd ed.). New York: McGraw-Hill, 1975.

SykES, R. E. Techniques of data collection and reduction in systematic field observation. Behavior Research Methods \& Instrumentation, 1977, 9, 407-417.

Wilson, M. C., Bellarosa, A. S., \& Bedford, J. A. Sociopharmacology of D-amphetamine in Macacca arctoides. Federation Proceedings, 1977, 36, 1039.

(Accepted for publication June 10, 1979.) 